\title{
Survival of patients with pancreatic cancer treated with varied modalities: A single-centre study
}

\author{
MICHAEL BLASZAK $^{1}$, MAHER EL-MASRI ${ }^{1,2}$, KHALID HIRMIZ ${ }^{1,3}$, JOHN MATHEWS ${ }^{1,3}$, ABEER OMAR ${ }^{2}$, \\ TAREK ELFIKI $^{1,3}$, RASNA GUPTA ${ }^{1,3}$, CAROLINE HAMM $^{1,3}$, SINDU KANJEEKAL $^{1,3}$, \\ AMIN KAY ${ }^{1,3}$, SWATI KULKARNI ${ }^{1,3}$ and AKMAL GHAFOOR ${ }^{1,3}$ \\ ${ }^{1}$ Schulich School of Medicine and Dentistry, Western University, London, ON N6A 5C1; ${ }^{2}$ Faculty of Nursing, \\ University of Windsor, Windsor, Ontario, ON N9B 3P4; ${ }^{3}$ Windsor Regional Hospital Cancer Program, \\ WRH Metropolitan Campus, Windsor, ON N8W 1L9, Canada
}

Received September 21, 2016; Accepted December 12, 2016

DOI: $10.3892 / \mathrm{mco} .2017 .1179$

\begin{abstract}
The present retrospective chart review examined the overall survival (OS) of patients with pancreatic ductal adenocarcinoma based on the disease stage in a sample of 296 patients with pancreatic cancer. Secondary outcome measurements included OS in chemotherapy vs. supportive treatment groups among metastatic patients, OS based on response to chemotherapy among metastatic patients, and OS and disease free survival (DFS) in surgically resected disease with vs. without adjuvant therapy. Data were analyzed using Kaplan-Meier and multivariate cox-regression analyses based on a $95 \%$ confidence interval (CI) or an $\alpha$-value of 0.05 . OS was significantly different based on the disease stage, with 3.63 (95\% CI, 2.84-4.43), 6.57 (95\% CI, 4.06-9.08) and 15.57 (95\% CI, 11.79-19.35) months in the advanced, locally advanced, and localized disease groups, respectively. OS was higher in metastatic-stage patients who received chemotherapy [6.07 months (95\% CI, 4.75-7.39)] compared with those who received supportive therapy alone $[2.50$ months $(95 \% \mathrm{CI}$, 2.16-2.84; $\mathrm{P}<.001)$ ]. Metastatic-stage patients with partial or stable response to chemotherapy had higher OS [10.53 months (95\% CI, 6.35-14.72)] in comparison with those with progression [6.33 months (95\% CI, 5.79-6.88)] or an undocumented response
\end{abstract}

Correspondence to: Dr Michael Blaszak, Schulich School of Medicine and Dentistry, Western University, 1151 Richmond Street, London, ON N6A 5C1, Canada

E-mail: mblaszak2016@meds.uwo.ca

Abbreviations: OS, overall survival; DFS, disease-free survival; CRT, chemoradiation therapy; Gem, gemcitabine; FOLFIRINOX, irinotecan-5-fluorouracil-leucoverin-oxaliplatin; 5FU, 5-fluorouracil; HR, hazard ratio; CI, confidence interval; ESPAC, European study group for pancreatic cancer; CT, computed tomography; ECOG, eastern cooperative oncology group; CA19-9, cancer antigen 19-9; RTOG, radiation therapy oncology group

Key words: pancreatic ductal adenocarcinoma, overall survival, disease free survival, chemotherapy, adjuvant therapy, prognosis, supportive therapy, surgical resection, margin, cancer
[3.30 months (95\% CI, 1.76-4.84; $\mathrm{P}<0.001)$ ]. In patients who underwent surgical resection of localized disease, adjuvant therapy increased the adjusted OS and DFS as compared with surgical excision alone ( $\mathrm{P}=0.013 ; 95 \% \mathrm{CI}, 0.278-0.862)$. Positive margins reduced OS [hazard ratio (HR) 2.670; 95\% CI, 1.467-4.860]. The present single-site study has demonstrated that OS may markedly differ on the basis of the disease status at the time of diagnosis. Metastatic-stage patients with stable or partial response to chemotherapy had an increased OS, as did surgical patients with localized disease who received adjuvant treatment, after adjusting for margin status.

\section{Introduction}

Pancreatic ductal adenocarcinoma is the fourth most common cancer-associated cause of death in the Western world (1). Surgical resection remains the only treatment modality with the potential for cure; however, only $15-20 \%$ of patients with pancreatic cancer are diagnosed with resectable disease (2). Given the high level of recurrences following surgery, both locally and systemically, adjuvant treatment using systemic chemotherapy, radiation and combined modalities has been used to improve the prospects of survival. However, the optimal treatment of choice remains controversial. Although findings of clinical trials have supported the use of adjuvant chemotherapy in patients with resected pancreatic cancer, reports are conflicting, particularly in the negative European Study Group for Pancreatic Cancer 1 (ESPAC-1) trial, concerning survival associated with the use of adjuvant chemoradiation therapy (CRT) (3).

Advanced pancreatic cancer is associated with an even worse prognosis; therefore, chemotherapies have been predominantly used to improve outcomes (4). Gemcitabine (Gem) has been the standard first-line chemotherapy since 1997 (5); however, two novel therapies, irinotecan-5-fluorouracil-leucoverin-oxaliplatin (FOLFIRINOX) and nab-paclitaxel plus Gem, have recently shown markedly improved overall survival (OS) rates in a certain subset of patients (6-9).

Given that the risk of pancreatic cancer increases with age, an aging population means that disease burden is expected to rise in the future. Thus, there is a clear need for further research on the diagnosis and treatment of pancreatic cancer (4). 
The primary aim of the present study was to explore the current OS of patients with pancreatic cancer treated at Windsor Regional Hospital Cancer Centre in southwestern Ontario, Canada, based on the stage of the disease. Secondary purposes of the study were to: (i) explore the OS of patients with metastatic pancreatic cancer treated with chemotherapy vs. supportive therapy alone; (ii) compare OS based on the response of these patients to chemotherapy, in order to determine whether patient response to therapy may help clinicians improve their predictions of patient prognosis; and (iii) to examine whether adjuvant therapy (chemotherapy, radiation or CRT) following the surgical resection of localized disease, as well as margin status, had any effect on disease-free survival (DFS) and OS.

\section{Patients and methods}

This study was a retrospective chart review of the medical records of 296 patients with pancreatic cancer treated at Windsor Regional Hospital Cancer Centre located in southwestern Ontario, Canada, between January 1, 2002 and May 9, 2013. The medical records of every pancreatic cancer patient treated at our regional cancer centre within the stated timeframe were reviewed via the online hospital database, and subsequently transferred to an Excel database after removing patient identifying information. All endocrine types, including intraductal papillary mucinous neoplasm, acinar cell carcinoma, cystadenocarcinoma, squamous cell carcinoma, and heptoid carcinoma of pancreatic origin, were excluded from the analysis due to the rarity of these tumors in the patient population under investigation. Histological pancreatic cancer diagnoses that were included in the present study consisted of adenocarcinoma, carcinoma only, carcinoma not otherwise specified (NOS) and signet ring cell carcinoma.

The date of diagnosis was defined as the date on which pathological evaluation of a tissue biopsy of either the primary tumour or metastasis confirmed malignancy. In cases where no tissue biopsy was performed (due to patient preference, patient health status and/or tumour location), but a clinical diagnosis of pancreatic cancer was noted in the chart, the date of diagnosis was instead defined as the date of the first computed tomography (CT) scan confirming the presence of a mass; these cases were specifically noted as having a 'radiological diagnosis only' during the later analysis. Cases where a tissue biopsy was performed, but a diagnosis could not be confirmed, were excluded.

Unless a date of mortality was recorded in the chart, the patient was listed as being alive at the date of last contact. Patients whose cause of death was unlikely to have been due to their diagnosis of pancreatic cancer were censored from the analysis, after the specific case had been reviewed by their respective oncologist.

The disease status (localized, locally advanced or metastatic/advanced) of the patient at the date of diagnosis was determined based on the documented clinician summary in the patient's chart; therefore, the patient's clinical presentation, radiological findings and histological evaluation were all taken into account.

Responsiveness to treatment was determined using the CT scan, with the date of recurrence or progression being defined as the date of the CT scan. Progression was defined as any radiologically confirmed increase in tumour size (in $\mathrm{cm}$ ) or further metastasis. In cases of multiple tumours in a patient, a decrease in the size of any tumour was defined as a partial response, regardless of growth of the remaining tumours. Recurrence was defined as radiological evidence of tumour grow th either locally or distally; patients with evidence of both local and distant recurrence were noted as 'distant recurrence'. Complete responsiveness to treatment was defined as the elimination of the tumour, as determined by the CT scan; however, there were no such cases in the present study.

The metastatic group included all patients with stage 4 cancer. Patients receiving any form of chemotherapy/CRT that was intended to have treated the pancreatic cancer after the date of diagnosis, regardless of whether they completed the chemotherapy regime, were placed into the 'chemotherapy group', whereas the rest were placed into the 'supportive therapy only' group for analysis. Chemotherapy regimens included primarily Gem or 5-fluorouracil (5FU), as well as a minority ( $\sim 11$ cases) of FOLFIRINOX or alternate combinations (5FU/Gem plus leucovorin, Gem plus 5FU, Gem plus erlotinib, or erlotinib alone).

Localized patients were defined as having either stage IA or IB disease at the time of diagnosis, based on the American Joint Committee on Cancer (AJCC) staging guidelines. Patients undergoing any surgical procedure with a curative intent were placed in the 'surgery' group, regardless of margin status post-procedure. Adjuvant therapy was defined as any chemotherapy or CRT administered post-surgical resection, and prior to any documented evidence of recurrence. CRT was given at either a dose of 45 Gray (Gy) in 25 fractions or 50.4 Gy in 28 fractions with concurrent bolus or infusion of 5FU-based chemotherapy. Margin status was determined based on the post-procedure pathology report, and classified into one of three categories: Positive, $\leq 1 \mathrm{~mm}$, and negative. The patient's response to treatment or recurrence status were listed as 'not documented' if the patient deteriorated rapidly, and no CT evaluation was performed to determine the response to treatment or recurrence prior to their date of mortality or the date of last contact.

Data analysis. Data were analyzed using the IBM SPSS version 22 (IBM SPSS, Armonk, NY, USA). Upon ensuring the accuracy of data entry and statistical assumptions, descriptive statistics were performed to describe the sample characteristics. Kaplan-Meier and multivariate Cox regression analyses were performed, as appropriate, to address the outlined study objectives.

Cox regression analysis of the metastatic population adjusted for general characteristics that were significantly different between groups. These included the patients' age, Eastern Cooperative Oncology Group (ECOG) status, and initial radiation treatment. Cox regression of the localized disease population also adjusted for margin status, as it was revealed to be significantly different between groups. All analyses were performed using a two-tailed $\alpha$-value of 0.05 , and either $\mathrm{P} \leq 0.05$ or the $95 \%$ confidence interval (CI) was considered to indicate a statistically significant value.

\section{Results}

Upon completion of the chart review, 18 of a total of 296 patients were deemed ineligible, yielding a sample of 278 patients 
Table I. Baseline characteristics divided into (A) the disease status and (B) the treatment type.

A, The disease status

\begin{tabular}{|c|c|c|c|c|c|c|}
\hline \multirow[b]{2}{*}{ Characteristic } & \multicolumn{3}{|c|}{ Disease status at diagnosis } & \multirow[b]{2}{*}{$\begin{array}{c}\text { Total } \\
(n=278)\end{array}$} & \multirow[b]{2}{*}{$\chi^{2} / F$} & \multirow[b]{2}{*}{ P-value } \\
\hline & $\begin{array}{l}\text { Localized } \\
\qquad(\mathrm{n}=93)\end{array}$ & $\begin{array}{l}\text { Locally advanced } \\
\qquad(\mathrm{n}=46)\end{array}$ & $\begin{array}{l}\text { Metastatic } \\
(n=139)\end{array}$ & & & \\
\hline Age $[$ mean $(\mathrm{SD})]$ & $66.08(11.90)$ & $67.04(11.94)$ & $66.45(12.88)$ & $66.42(12.73)$ & 0.09 & 0.91 \\
\hline Gender $[\mathrm{n},(\%)]$ & & & & & 0.09 & 0.95 \\
\hline Male & $51(54.8)$ & $26(56.5)$ & $79(56.8)$ & $156(56.1)$ & & \\
\hline Female & $42(45.2)$ & $20(43.5)$ & $60(43.2)$ & $122(43.9)$ & & \\
\hline ECOG score at presentation $[\mathrm{n},(\%)]$ & & & & & 3.66 & 0.45 \\
\hline $0-2$ & $58(62.4)$ & $25(54.3)$ & $86(61.9)$ & $169(60.8)$ & & \\
\hline $3-4$ & $11(11.8)$ & $6(13.0)$ & $24(17.3)$ & $41(14.7)$ & & \\
\hline Not documented & $24(25.8)$ & $15(32.6)$ & $29(20.9)$ & $68(24.5)$ & & \\
\hline
\end{tabular}

$\mathrm{B}$, The treatment type

\begin{tabular}{|c|c|c|c|c|c|}
\hline \multirow[b]{2}{*}{ Characteristic } & \multicolumn{2}{|c|}{ Group } & \multirow[b]{2}{*}{ Total $(n=213)$} & \multirow[b]{2}{*}{$\chi^{2 / t}$} & \multirow[b]{2}{*}{ P-value } \\
\hline & Supportive $(\mathrm{n}=78)$ & Chemo $(n=61)$ & & & \\
\hline Age [mean (SD)] & $69.47(12.89)$ & $62.59(11.89)$ & & 3.32 & $<0.01$ \\
\hline Gender [n, (\%)] & & & & 1.23 & 0.25 \\
\hline Male & $41(52.6)$ & $38(62.3)$ & $79(56.8)$ & & \\
\hline Female & $37(47.4)$ & $23(37.7)$ & $60(43.2)$ & & \\
\hline ECOG score at presentation $[\mathrm{n},(\%)]$ & & & & 12.10 & $<0.01$ \\
\hline $0-2$ & $41(52.6)$ & $45(73.8)$ & $86(61.9)$ & & \\
\hline $3-4$ & $21(26.9)$ & $3(4.9)$ & $24(17.3)$ & & \\
\hline Not documented & $16(20.5)$ & $13(21.3)$ & $29(20.9)$ & & \\
\hline Initial radiation treatment $[\mathrm{n},(\%)]$ & & & & 0.44 & 0.51 \\
\hline None & $72(92.3)$ & $58(95.1)$ & $130(93.5)$ & & \\
\hline Palliative & $6(7.7)$ & $3(4.9)$ & $9(605)$ & & \\
\hline
\end{tabular}

ECOG, eastern cooperative oncology group.

(93 localized, 46 locally advanced and 139 metastatic). The baseline characteristics of age, gender, and ECOG status were not significantly different between the groups, as shown in Table I. The mean age at diagnosis was 66 years [standard deviation (SD) \pm 12.73 ]. The majority of patients presented with metastatic disease (50\%) and an ECOG between 0-2 (61\%). A total of 80 patients $(29 \%)$ presented with localized disease that was treated with surgical resection.

Table IB shows that the chemotherapy and supportive therapy groups for patients with metastatic cancer were significantly different in their age and ECOG score $(\mathrm{P}<0.01)$. The median OS was identified to be statistically significant $(\mathrm{P}<0.001)$ among groups, with 3.63 (95\% CI, 2.84-4.43) months in the metastatic group, 6.57 (95\% CI, 4.06-9.08) months in the locally advanced group, and 15.57 (95\% CI, 11.79-19.35) months in the localized disease group (Table II). The results of the Kaplan-Meier analysis indicated that the median OS was significantly longer in metastatic patients who received chemotherapy [6.07 months (95\% CI, 4.75-7.39)] compared with those who received supportive therapy alone [2.50 months (95\% CI, 2.16-2.84; P<0.001)] (Table II). This result held true in the multivariate cox regression analysis, after adjusting for age, ECOG status and initial radiation treatment (Table III).

The results pertaining to the subgroup of metastatic patients receiving chemotherapy (Table II) indicated that those with a partial or stable response to treatment had a significantly higher median OS [10.53 months (95\% CI, 6.35-14.72)] compared with those with progression [6.33 months (95\% CI, 5.79-6.88)] or an undocumented response [3.30 months (95\% CI, 1.76-4.84); $\mathrm{P}<0.001]$. Within the subgroup of patients who underwent surgical resection (Tables IV and V), adjuvant treatment was not associated with an increase in the median OS $(\mathrm{P}=0.09)$, DFS $(\mathrm{P}=0.60)$ or recurrence location $(\mathrm{P}=0.14)$. However, adjuvant treatment significantly increased the median $\mathrm{OS}$ after adjusting for the margin and nodal status ( $\mathrm{P}=0.013$; 95\% CI, 0.278-0.862). Once the margin status had been adjusted, patients with 
Table II. Kaplan-Meier survival comparisons.

\begin{tabular}{lcc}
\hline Group & $\begin{array}{c}\text { Sample } \\
\text { size }(\mathrm{n})\end{array}$ & $\begin{array}{c}\text { Median survival in } \\
\text { month }(95 \% \text { CI })\end{array}$ \\
\hline Overall & 278 & $15.57(11.79-19.35)$ \\
Localized disease & 93 & $3.63(2.84-4.43)$ \\
Metastatic & 139 & $6.57(4.06-9.08)$ \\
Locally advanced & 46 & \\
Metastatic group & 139 & $6.07(4.75-7.39)$ \\
Chemotherapy treatment & 61 & $2.50(2.16-2.84)$ \\
Supportive treatment & 78 & $10.53(6.35-14.72)$ \\
Response to chemotherapy in metastatic group & 61 & $6.33(5.79-6.88)$ \\
Partial or stable response & 10 & $3.30(1.76-4.84)$ \\
Progression & 23 & $18.80(15.54-22.15)$ \\
Not documented & 28 & $10.53(3.83-17.23)$
\end{tabular}

CI, confidence interval.

Table III. Cox regression comparing overall survival based on type of treatment among metastatic disease patients.

\begin{tabular}{|c|c|c|c|c|c|}
\hline Variable & $\beta$-value & SE & P-value & Hazard ratio & $95 \% \mathrm{CI}$ \\
\hline Age (years) & 0.01 & 0.01 & 0.56 & 1.01 & 0.99-1.02 \\
\hline Gender (male) & 0.48 & 0.20 & 0.02 & 1.16 & $1.10-2.38$ \\
\hline Treatment (chemo) & -0.75 & 0.21 & $<0.01$ & 0.47 & $0.32-0.70$ \\
\hline Supportive (reference group) & - & - & - & - & - \\
\hline ECOG 3-4 & 0.14 & 0.27 & 0.61 & 1.15 & $0.68-1.94$ \\
\hline ECOG not documented & 0.11 & 0.26 & 0.67 & 1.12 & $0.67-1.85$ \\
\hline ECOG 0-2 (Reference) & - & - & - & - & - \\
\hline Initial radiation treatment & -0.24 & 0.40 & 0.55 & 0.79 & $0.36-1.71$ \\
\hline
\end{tabular}

ECOG, Eastern cooperative oncology group; SE, standard error.

positive margins were revealed to have significantly shorter OS [hazard ratio $(\mathrm{HR})=2.670 ; 95 \% \mathrm{CI}, 1.467-4.860]$. Intermediate margins had no significant effect on OS (HR=1.429; 95\% CI, 0.566-3.605). Positive lymph node status was not associated with decreased survival in the $1-2$ positive node group $(\mathrm{P}=0.097)$, or in the $3+$ positive node group $(\mathrm{P}=0.106)$. Table $\mathrm{V}$ shows that 18 of 80 patients $(22 \%)$ with surgical resection did not have any documented recurrence of disease.

\section{Discussion}

Clinical trials have supported the use of adjuvant chemotherapy in patients with resected pancreatic cancer $(10,11)$. However, the use of CRT in adjuvant treatment remains a hotly debated topic, and currently no studies have been published demonstrating the superiority of the addition of radiotherapy with modern delivery techniques to adjuvant chemotherapy alone $(2,12,13)$. Furthermore, efforts are now focused on increasing the resectability of locoregional disease by incorporating neoadjuvant treatment prior to surgery. Adjuvant CRT is, however, generally agreed upon to improve local control in surgically resected patients with adverse risk factors for locoregional disease recurrence that include an older age, large tumour size, advanced tumour stage, high histological grade, an elevated cancer antigen 19-9 (CA19-9) level, positive lymph nodes and positive surgical margins (13).

In this single-centre chart review, a higher percentage of patients $(29 \%)$ were diagnosed with localized disease, and were subsequently treated with surgical resection, compared with the previously cited value of $15-20 \%$ (2). Furthermore, patients treated with adjuvant therapy (chemotherapy or CRT) had a median OS that was comparable with previous studies, which ranged from 17.1-23.2 months $(10,11,14,15)$. It is important to note, however, that these published studies excluded certain patients based on various negative prognostic factors [i.e., the CONKO-001 trial excluded patients 
Table IV. Cox regression comparing survival based on adjuvant treatment among localized pancreatic cancer patients undergoing resection.

\begin{tabular}{|c|c|c|c|c|c|}
\hline Variable & $\beta$-value & SE & P-value & Hazard ratio & $95 \% \mathrm{CI}$ \\
\hline Adjuvant treatment & -0.715 & 0.289 & 0.013 & 0.489 & $0.278-0.862$ \\
\hline \multicolumn{6}{|l|}{ Margin status } \\
\hline Positive & 0.982 & 0.306 & 0.001 & 2.670 & $1.467-4.860$ \\
\hline$\leq 1 \mathrm{~mm}$ & 0.357 & 0.472 & 0.450 & 1.429 & $0.566-3.605$ \\
\hline Negative (reference) & - & - & - & - & - \\
\hline \multicolumn{6}{|l|}{ Node status } \\
\hline 3 or more positive nodes & 0.563 & 0.348 & 0.106 & 1.755 & $0.888-3.470$ \\
\hline 1-2 positive nodes & 0.606 & 0.365 & 0.097 & 1.833 & $0.897-3.745$ \\
\hline Negative nodes (reference) & - & - & - & - & - \\
\hline
\end{tabular}

CI, confidence interval.

Table V. Disease-free survival and recurrence status of localized disease patients undergoing surgical resection.

\begin{tabular}{|c|c|c|c|c|c|}
\hline \multirow[b]{2}{*}{ Variable } & \multicolumn{2}{|c|}{ Group } & \multirow[b]{2}{*}{ Total $(\mathrm{n}=80)$} & \multirow[b]{2}{*}{$\chi^{2 / t}$} & \multirow[b]{2}{*}{ P-value } \\
\hline & Adjuvant $(\mathrm{n}=52)$ & No adjuvant $(n=28)$ & & & \\
\hline Recurrence time in months [mean, (SD)] & $13.39(13.43)$ & $11.47(11.42)$ & $12.73(12.70)$ & -0.53 & 0.60 \\
\hline \multicolumn{6}{|l|}{ Recurrence status [n, (\%)] } \\
\hline None & $14(26.9)$ & $4(14.3)$ & $18(22.5)$ & & \\
\hline Local/regional only & $5(9.6)$ & $3(10.7)$ & $8(10.0)$ & 5.42 & 0.14 \\
\hline Distant & $31(59.6)$ & $16(57.1)$ & $47(58.7)$ & & \\
\hline Not documented & $2(3.9)$ & $5(17.9)$ & $7(8.8)$ & & \\
\hline
\end{tabular}

with CA19-9 levels $>2.5$ times the upper limit of normal, the ESPAC-1 and ESPAC-3 trials excluded patients with macroscopically remaining tumour post-resection, and the Radiation Therapy Oncology Group (RTOG) trial excluded patients with lower performance scores, which limits the comparison]. Furthermore, in the subgroup of patients who were treated with surgical resection alone, the fact that patients were not excluded based on negative prognostic criteria in the present study provides a possible explanation for why the median OS was comparatively shorter than the range of 11-20.2 months that was reported by Sen et al (2).

In contrast with previous studies, which reported that positive nodal status is a negative prognostic indicator, the present study identified no significant effect of nodal status on prognosis (13). A possible explanation of this discrepancy could be attributed to the lack of standardization among patients with regard to the number of lymph nodes that were sent to pathology for evaluation (ranging from 0 to $>3$ ) in the present study, whereas, by contrast, the RTOG trial by Regine et al (15) did standardize for the number of lymph nodes, and had that as part of their eligibility criteria. It is important to note, however, that standardization in the present study was not possible, given its retrospective nature.

The present study is the first, to the best of our knowledge, that has explored intermediate margin status, and the findings in this study indicated no difference in the median OS between patients with intermediate margins and those with negative margins. This lack of difference in the median OS suggests that these patients may not be at an increased risk, and thus will not benefit from CRT as long as they do not have any other adverse risk factors for locoregional disease recurrence. Consistent with several published reports $(10,11,13,16,17)$, the findings of the present study suggest that positive margin status is a negative prognostic indicator, further demonstrating that margin status is independently associated with OS. However, two previously published studies $(10,11)$ suggested that margin status is of significantly less prognostic value than primary tumour characteristics, which tend to dominate the outcome.

The findings of the present study have shown that patients with metastatic pancreatic cancer who received chemotherapy had a significantly longer median OS. This finding was consistent with the findings of past studies that reported on patients with advanced stage pancreatic cancer who were treated with single-agent Gem, as well as Gem plus other cytotoxic and targeted agents (1). Furthermore, a previous study (8) suggested that OS is also influenced by a number of independent, adverse prognostic factors, including age and gender. In contrast with the present study, Moore et al (18) and Conroy et al (8) reported that age was negatively associated with OS. The reasons for this discrepancy are not entirely clear, although it is possible that 
it may be a result of differences in the patient sample size, i.e., in our study there were 139 patients with metastatic disease, compared with 569 and 342 in the above-mentioned articles respectively. Therefore, it is advisable that a further study should investigate the association between age and OS with a larger sample size, to reduce the possibility of type II error. The present study has suggested that response to therapy, in patients with metastatic disease who are treated with chemotherapy, is potentially a beneficial prognostic factor.

Di Marco et al (1) argued that Gem remains a mainstay of treatment for advanced pancreatic cancer, and that the combination of Gem with a variety of cytotoxic and targeted agents has generally revealed no significant survival advantage as compared with Gem alone, aside from the recent metastatic adenocarcinoma of the pancreas (MPACT) study by Tabernero et al (9). The present study examined the OS of patients treated with chemotherapy, and did not compare specific treatment regimes, since, in this study, the majority of patients were treated with Gem alone. In recent years, FOLFIRINOX has emerged as the most effective chemotherapy regime in a select subset of patients with pancreatic cancer (8). Given that Windsor Regional Hospital Cancer Centre has recently begun to treat more patients with FOLFIRINOX, further research is warranted to determine whether patients' OS improves in response to the introduction of FOLFIRINOX.

It is important to note that the findings in the present study need to be interpreted with caution due to the retrospective chart review nature of our study, which increases the potential for confounding, selection and measurement bias. The relatively small sample size that was obtained from a single community-based cancer centre presents an additional limitation to this study that ought to be taken in consideration prior to making generalizations based on the findings. As the centre's patient sample increases in size, further studies evaluating adjuvant CRT against adjuvant chemotherapy alone may provide useful information to add to the current debate.

In conclusion, in the present study OS was revealed to significantly differ on the basis of the disease status at diagnosis, with patients diagnosed with localized disease having the longest OS. Furthermore, in metastatic patients receiving chemotherapy, a partial or stable response to chemotherapy appeared to significantly increase OS in comparison with those with progression. Adjuvant therapy significantly increased OS in comparison with patients treated with surgical excision alone, once margin and nodal status had been adjusted for. Positive margins were a negative prognostic indicator. Localized patients undergoing surgical resection treated with adjuvant therapy were identified to have significantly longer OS after adjusting for margin status, with positive margins significantly reducing survival; however, intermediate margins were not associated with a significant difference in OS when compared with negative margins.

This study has added to the burgeoning body of evidence that will aid clinicians in terms of determining prognostic estimates during the treatment of pancreatic ductal adenocarcinoma.

\section{References}

1. Di Marco M, Di Cicilia R, Macchini M, Nobili E, Vecchiarelli S, Brandi $G$ and Biasco G: Metastatic pancreatic cancer: Is gemcitabine still the best standard treatment? (Review). Oncol Rep 23: 1183-1192, 2010.
2. Sen N, Falk S and Abrams R: Role of chemoradiotherapy in the adjuvant and neoadjuvant settings for resectable pancreatic cancer. Clin Oncol (R Coll Radiol) 26: 551-559, 2014.

3. Seufferlein T, Bachet JB, Van Cutsem E and Rougier P; ESMO Guidelines Working Group: Pancreatic adenocarcinoma: ESMO-ESDO Clinical Practice Guidelines for diagnosis, treatment and follow-up. Ann Oncol 23 (Suppl 7): vii33-vii40, 2012.

4. Preis $\mathrm{M}$ and Korc M: Kinase signaling pathways as targets for intervention in pancreatic cancer. Cancer Biol Ther 9: 754-763, 2010.

5. Kleger A, Perhofer L and Seufferlein T: Smarter drugs emerging in pancreatic cancer therapy. Ann Oncol 25: 1260-1270, 2014.

6. Vaccaro V, Sperduti I and Milella M: FOLFIRINOX versus gemcitabine for metastatic pancreatic cancer. N Engl J Med 365: 768-769, 2011.

7. Gourgou-Bourgade S, Bascoul-Mollevi C, Desseigne F, Ychou M, Bouché O, Guimbaud R, Bécouarn Y, Adenis A, Raoul JL, Boige V, et al: Impact of FOLFIRINOX compared with gemcitabine on quality of life in patients with metastatic pancreatic cancer: Results from the PRODIGE 4/ACCORD 11 randomized trial. J Clin Oncol 31: 23-29, 2013.

8. Conroy T, Desseigne F, Ychou M, Bouché O, Guimbaud R, Bécouarn Y, Adenis A, Raoul JL, Gourgou-Bourgade S, de la Fouchardière $\mathrm{C}$, et al: FOLFIRINOX versus gemcitabine for metastatic pancreatic cancer. N Engl J Med 364: 1817-1825, 2011.

9. Tabernero J, Chiorean EG, Infante JR, Hingorani SR, Ganju V, Weekes C, Scheithauer W, Ramanathan RK, Goldstein D, Penenberg DN, et al: Prognostic factors of survival in a randomized phase III trial (MPACT) of weekly nab-paclitaxel plus gemcitabine versus gemcitabine alone in patients with metastatic pancreatic cancer. Oncologist 20: 143-150, 2015.

10. Neoptolemos JP, Stocken DD, Friess H, Bassi C, Dunn JA, Hickey $\mathrm{H}$, Beger $\mathrm{H}$, Fernandez-Cruz L, Dervenis C, Lacaine F, et al: A randomized trial of chemoradiotherapy and chemotherapy after resection of pancreatic cancer. N Engl J Med 350: 1200-1210, 2004

11. Neoptolemos JP, Stocken DD, Bassi C, Ghaneh P, Cunningham D, Goldstein D, Padbury R, Moore MJ, Gallinger S, Mariette C, et al: Adjuvant chemotherapy with fluorouracil plus folinic acid vs gemcitabine following pancreatic cancer resection: A randomized controlled trial. JAMA 304: 1073-1081, 2010.

12. Miller RC, Iott MJ and Corsini MM: Review of adjuvant radiochemotherapy for resected pancreatic cancer and results from Mayo Clinic for the 5th JUCTS symposium. Int J Radiat Oncol Biol Phys 75: 364-368, 2009.

13. Chuong MD, Boggs DH, Patel KN and Regine WF: Adjuvant chemoradiation for pancreatic cancer: What does the evidence tell us? J Gastrointest Oncol 5: 166-177, 2014.

14. Ouettle H, Neuhaus P, Hochhaus A, Hartmann JT, Gellert K, Ridwelski K, Niedergethmann M, Zülke C, Fahlke J, Arning MB, et al: Adjuvant chemotherapy with gemcitabine and long-term outcomes among patients with resected pancreatic cancer: The CONKO-001 randomized trial. JAMA 310: 1473-1481, 2013.

15. Regine WF, Winter KA, Abrams R, Safran H, Hoffman JP, Konski A, Benson AB, Macdonald JS, Rich TA and Willett CG: Fluorouracil-based chemoradiation with either gemcitabine or fluorouracil chemotherapy after resection of pancreatic adenocarcinoma: 5-year analysis of the U.S. Intergroup/RTOG 9704 phase III trial. Ann Surg Oncol 18: 1319-1326, 2011.

16. Moghanaki D, Mick R, Furth EE, Sohal D, Salmon PM, Behbahani A, Morgans AK, Miller SM, Giantonio BJ, Whittington RW, et al: Resection status, age and nodal involvement determine survival among patients receiving adjuvant chemoradiotherapy in pancreatic adenocarcinoma. JOP 12: 438-444, 2011.

17. Herman JM, Swartz MJ, Hsu CC, Winter J, Pawlik TM, Sugar E, Robinson R, Laheru DA, Jaffee E, Hruban RH, et al: Analysis of fluorouracil-based adjuvant chemotherapy and radiation after pancreaticoduodenectomy for ductal adenocarcinoma of the pancreas: Results of a large, prospectively collected database at the Johns Hopkins Hospital. J Clin Oncol 26: 3503-3510, 2008.

18. Moore MJ, Goldstein D, Hamm J, Figer A, Hecht JR, Gallinger S, $\mathrm{Au} \mathrm{HJ}$, Murawa P, Walde D, Wolff RA, et al: Erlotinib plus gemcitabine compared with gemcitabine alone in patients with advanced pancreatic cancer: A phase III trial of the National Cancer Institute of Canada Clinical Trials group. J Clin Oncol 25: 1960-1966, 2007. 